\title{
A QUEUEING MODEL OF FINITE AND INFINITE SOURCE INTERACTION
}

\author{
O.J. BOXMA \\ Centre for Mathematics and Computer Science, P.O. Box 4079, 1009 A B Amsterdam, The Netherlands
}

Received October 1985

Revised September 1986

An $M / M / 1$ service system is considered, which also serves one finite source. The joint distribution of queue length at the $M / M / 1$ queue and position of the finite source customer in the system is determined. This leads to exact expressions for various performance measures; such expressions yield insight into the interaction of finite and infinite sources.

$M / M / 1$ queue $*$ finite source $*$ interaction

\section{Introduction}

Consider the following simple model of finite and infinite source interaction. Infinite source customers arrive at a single server queue $Q$ according to a stationary Poisson process with rate $\lambda$, requiring negative exponentially distributed service times with mean $1 / \mu_{\mathrm{p}}$. There is one finite source (fs), having negative exponentially distributed think times with mean $1 / \gamma$. The finite source customer requires at $Q$ a negative exponentially distributed service time with mean $1 / \mu_{\mathrm{fs}}$. Both customer types thus share $Q$ (cf. Figure 1 ). The quenching discipline at $Q$ is FCFS; hence no customer type has priority over the other type.

This model, and its generalization to the case of $N$ identical finite sources, has been studied by Kaufman [2]. He points out that, if $\mu_{\mathrm{P}}=\mu_{\mathrm{fs}}$, the model is a simple product-form network, whereas if $\mu_{\mathrm{P}} \neq \mu_{\mathrm{fs}}$, the resulting model is no longer product-form and in fact appears to be intractable'. Subsequently he presents a very accurate approximation method for estimating all mean performance measures of interest, thus obtaining insight in the extent to which the fs (finite source) customer increases the congestion experienced by the Poisson customers.

The main goal of the present paper is to give an exact analysis of the model described above, with one finite source. In Section 2 we obtain an explicit expression for the generating function of the joint distribution of queue length at $Q$ and position of the fs customer in the system. From this generating function, exact formulas for all mean performance measures of interest are derived. In Section 3 we study the asymptotic behavior of the queue length at $Q$, thus obtaining some extra insight into the interaction of

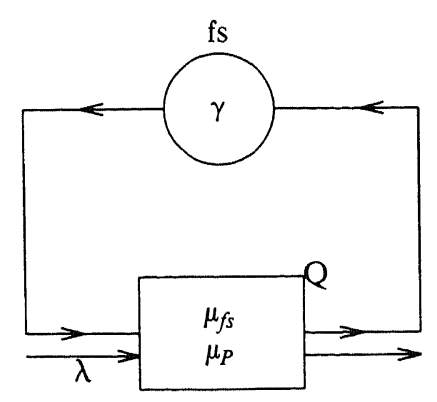

Fig. 1. The interaction model. 
the finite and infinite source customers - an interaction about which not much is known in non-productform networks.

In the sequel we assume that

$$
\rho:=\frac{\lambda}{\mu_{\mathrm{P}}}<1
$$

It is clear from $M / M / 1$ theory that this is a necessary condition for ergodicity of the system. It is not difficult to see that this condition is also sufficient. One can in fact extend the analysis of Section 2 to the time-dependent case, and use the thus obtained results to show the sufficiency of condition (1.1); but heuristically it is quite clear that, with FCFS Service in $Q$, the one fs customer should not be able to influence the ergodicity of the $M / M / 1$ queue.

\section{Remark concerning literature}

The method employed in the solution of the model is similar to the method used by Takacs [3] to analyse a model of two queues with alternating priority. The model is similar to one discussed by, i.a., Vinod and Solberg [4], the only difference being that in the model of [4] the fs customer has preemptive-resume priority over the Poisson customers - a fact which strongly influences the mathematical analysis. In their model $Q$ represents a machine that can break down, after which it is repaired by the fs customer (who hence clearly has priority).

\section{Analysis}

Let

$$
h(x, m):=\operatorname{Pr}\{\boldsymbol{x}=x, \boldsymbol{n}=x+m\}, \quad x, m=0, \overline{1}, \ldots,
$$

with

$\boldsymbol{n}:=$ the total number of customers in $Q$,

$x:=$ the position of the fs customer;

here

$\boldsymbol{x}=0$ if the fs customer is in its source,

$\boldsymbol{x}=i$ if the fs customer is in $Q$ in position $i$ (position 1 denoting: in service).

Then,

$$
\begin{aligned}
& \left(\lambda+\mu_{\mathrm{P}}\right) h(x, m)=\lambda h(x, m-1)+\mu_{\mathrm{p}} h(x+1, m), \quad x=2,3, \ldots, \quad m=1,2, \ldots, \\
& \left(\lambda+\mu_{\mathrm{fs}}\right) h(1, m)=\lambda h(1, m-1)+\mu_{\mathrm{p}} h(2, m), \quad m=1,2, \ldots, \\
& \left(\lambda+\mu_{\mathrm{p}}\right) h(x, 0)=\gamma h(0, x-1)+\mu_{\mathrm{p}} h(x+1,0), \quad x=2,3, \ldots, \\
& \left(\lambda+\mu_{\mathrm{fs}}\right) h(1,0)=\gamma h(0,0)+\mu_{\mathrm{p}} h(2,0), \\
& \left(\lambda+\mu_{\mathrm{p}}+\gamma\right) h(0, m)=\lambda h(0, m-1)+\mu_{\mathrm{p}} h(0, m+1)+\mu_{\mathrm{fs}} h(1, m), \quad m=1,2, \ldots, \\
& (\lambda+\gamma) h(0,0)=\mu_{\mathrm{p}} h(0,1)+\mu_{\mathrm{fs}} h(1,0) .
\end{aligned}
$$

Introducing

$$
\begin{aligned}
& H(w, z):=\sum_{x=0}^{\infty} \sum_{m=0}^{\infty} h(x, m) w^{x} z^{m}, \quad|w| \leqslant 1, \quad|z| \leqslant 1, \\
& H_{1}(z):=\sum_{m=0}^{\infty} h(1, m) z^{m}, \quad|z| \leqslant 1,
\end{aligned}
$$


it follows from (2.2a)-(2.2f) and (2.2e), (2.2f), respectively, that for $|w| \leqslant 1,|z| \leqslant 1$,

$$
\begin{aligned}
{\left[\lambda(1-z)+\mu_{\mathrm{P}}\left(1-\frac{1}{w}\right)\right] H(w, z)=} & \left(\mu_{\mathrm{P}}-\mu_{\mathrm{fs}}\right)(w-1) H_{1}(z)+\mu_{\mathrm{P}}\left(1-\frac{1}{z}\right) H(0,0) \\
& +\gamma w H(0, w)+\left(\frac{\mu_{\mathrm{P}}}{z}-\frac{\mu_{\mathrm{P}}}{w}-\gamma\right) H(0, z), \\
H_{1}(z)=\frac{1}{\mu_{\mathrm{fs}}}\left[\lambda(1-z)+\mu_{\mathrm{P}}\left(1-\frac{1}{z}\right)\right. & +\gamma] H(0, z)-\frac{\mu_{\mathrm{P}}}{\mu_{\mathrm{fs}}}\left(1-\frac{1}{z}\right) H(0,0) .
\end{aligned}
$$

Substitution of (2.6) into (2.5) yields after some calculations

$$
\begin{aligned}
& H(w, z)-H(0, z) \\
& \quad=\frac{\gamma w H(0, w)+\left[\lambda(1-z)+\mu_{\mathrm{P}}(1-1 / z)+\gamma\right]\left[\left(\mu_{\mathrm{P}} / \mu_{\mathrm{fs}}\right)(w-1)-w\right] H(0, z)}{\lambda(1-z)+\mu_{\mathrm{P}}(1-1 / w)} \\
& \quad-\frac{\mu_{\mathrm{P}}(1-1 / z)\left[\left(\mu_{\mathrm{P}} / \mu_{\mathrm{fs}}\right)(w-1)-w\right] H(0,0)}{\lambda(1-z)+\mu_{\mathrm{P}}(1-1 / w)}, \quad|w| \leqslant 1, \quad|z| \leqslant 1 .
\end{aligned}
$$

Once $H(0, z)$ is determined for $|z| \leqslant 1, H(w, z)$ follows from (2.7). Determination of $H(0, z)$ proceeds as follows. The denominator of the rhs. of (2.7) becomes zero for

$$
w=\delta(z):=\left[1+\frac{\lambda}{\mu_{\mathrm{P}}}(1-z)\right]^{-1} .
$$

For $|z| \leqslant 1$, clearly $|\delta(z)| \leqslant 1$ (in fact, $\delta(z)$ is the LST of the service time distribution of the Poisson customers, evaluated at $\lambda(1-z)$. Since the lhs. of (2.7) is analytic in $|w| \leqslant 1,|z| \leqslant 1$, the numerator of the rhs, of (2.7) should be zero for $w=\delta(z),|z| \leqslant 1$. So for all $z$ with $|z| \leqslant 1$,

$$
H(0, z)=C(z) H(0,0)+D(z) H(0, \delta(z)) \text {, }
$$

with

$$
\begin{aligned}
& C(z):=\frac{\mu_{\mathrm{p}}(1-1 / z)}{\lambda(1-z)+\mu_{\mathrm{p}}(1-1 / z)+\gamma}, \\
& D(z):=\frac{\gamma}{\lambda(1-z)+\mu_{\mathrm{p}}(1-1 / z)+\gamma} \frac{\mu_{\mathrm{fs}}}{\lambda(1-z)+\mu_{\mathrm{fs}}} .
\end{aligned}
$$

Note that $C(z)$ and $D(z)$ have exactly one pole $z_{1}, 0<z_{1}<1$, in $|z| \leqslant 1$,

$$
z_{1}=\left[\lambda+\mu_{\mathrm{P}}+\gamma-\sqrt{\left(\lambda+\mu_{\mathrm{P}}+\gamma\right)^{2}-4 \lambda \mu_{\mathrm{P}}}\right] / 2 \lambda \text {. }
$$

Remark 1. In the extreme case $\gamma=0$ (the fs customer never leaves its source) it follows from (2.7) and (2.9), (2.10), (2.11) that

$$
H(w, z)=H(0, z)=\frac{\mu_{\mathrm{p}}}{\mu_{\mathrm{p}}-\lambda z} H(0,0), \quad|z| \leqslant 1,
$$

which indeed leads to standard $M / M / 1$ results.

We have to determine $H(0, z)$ from the conditions that, for all $z$ with $|z| \leqslant 1$, (i) it is an analytic function of $z$, and (ii) it satisfies (2.9). $\delta(z)$ and its iterates, defined below, play an essential role in this analysis. Define, for $|z| \leqslant 1$,

$$
\begin{aligned}
& \delta^{(0)}(z):=z, \\
& \delta^{(n)}(z):=\delta\left(\delta^{(n-1)}(z)\right), \quad n=1,2, \ldots
\end{aligned}
$$

For future reference we collect some results concerning $\delta^{(n)}(z)$ in the following lemma. 
Lemma 1. For $|z| \leqslant 1, n=1,2, \ldots$,

$$
\begin{aligned}
& \text { (i) } \delta^{(n)}(z)=1-\rho^{n} \frac{1-z}{1+(1-z)\left(\left(\rho-\rho^{n+1}\right) /(1-\rho)\right)}, \\
& \text { (ii) } \frac{1-\delta^{(n)}(z)}{1-\delta^{(n-1)}(z)}=\rho \delta^{(n)}(z) \\
& \text { (iii) }\left|\delta^{(n)}(z)\right| \leqslant 1 \\
& \text { (iv) }\left|\frac{1-\delta^{(n)}(z)}{1-\delta^{(n-1)}(z)}\right|<1 \\
& \text { (v) } \lim _{n \rightarrow \infty} \delta^{(n)}(z)=1
\end{aligned}
$$

Proof. Trivial.

Remark 2. It follows from (2.10), (2.11) and Lemma 1(i) that for $n \rightarrow \infty$,

$$
C\left(\delta^{(n)}(z)\right) \sim \rho^{n}, \quad 1-D\left(\delta^{(n)}(z)\right) \sim \rho^{n} .
$$

Iterating relation (2.9) we obtain (an empty product being one, by definition)

$$
H(0, z)=H(0,0) \sum_{i=0}^{\infty}\left\{C\left(\delta^{(i)}(z)\right) \prod_{j=0}^{i-1} D\left(\delta^{(j)}(z)\right)\right\}+H(0,1) \prod_{j=0}^{\infty} D\left(\delta^{(j)}(z)\right), \quad|z| \leqslant 1 .
$$

We shall now determine the two unknowns $H(0,0)$ and $H(0,1)$ in $(2.15)$. One relation between $H(0,0)$ and $H(0,1)$ is obtained by observing that $H(0, \mathrm{z})$ should be analytic in $|z| \leqslant 1$. Using Lemma 1 and (2.14) one can show that $\prod_{j=0}^{\infty} D\left(\delta^{(j)}(z)\right)$ and $\sum_{i=0}^{\infty}\left\{C\left(\delta^{(i)}(z)\right) \prod_{j=0}^{i-1} D\left(\delta^{(j)}(z)\right)\right\}$ are well-defined and analytic in $|z| \leqslant 1$, except for those $z$ for which a non-negative integer $n$ exists such that $\delta^{(n)}(z)=z_{1}$ (cf. (2.12)); we omit the details. In particular, $D(z)$, occurring in every term in the right-hand side of (2.15), has a pole at $z=z_{1}$. Divide both sides of $(2.15)$ by $D(z)$, and subsequently put $z=z_{1}$; the analyticity of $H(0, \mathrm{z})$ in $z=z_{1}$ now implies that

$$
H(0,0)\left[\sum_{i=1}^{\infty}\left\{C\left(\delta^{(i)}\left(z_{1}\right)\right) \prod_{j=1}^{i-1} D\left(\delta^{(j)}\left(z_{1}\right)\right)\right\}+\frac{C\left(z_{1}\right)}{D\left(z_{1}\right)}\right]+H(0,1) \prod_{j=1}^{\infty} D\left(\delta^{(j)}\left(z_{1}\right)\right)=0 .
$$

Here, by definition,

$$
\frac{C\left(z_{1}\right)}{D\left(z_{1}\right)}=\mu_{\mathrm{P}}\left(1-\frac{1}{z_{1}}\right) \frac{1}{\gamma}\left(1+\frac{\lambda}{\mu_{\mathrm{fs}}}\left(1-z_{1}\right)\right)
$$

cf. (2.10) and (2.11).

Similarly, divide both sides of $(2.15)$ by $D(z) D(\delta(z))$, and put $z=\delta^{-1}\left(z_{1}\right)$; the analyticity of $H(0, z)$ in $z=\delta^{-1}\left(z_{1}\right)$ again implies relation (2.16). Continuing in the same way, it is seen that condition (2.16) ensures the analyticity of $H(0, z)$ at all those values of $z$, for which a positive integer $n$ exists such that $\delta^{(n)}(z)=z_{1}$ (the fact that one and the same condition takes care of all the singularities is a direct consequence of the structure of $(2.9)$ and of $C(\cdot)$ and $D(\cdot))$.

Relation (2.16) provides one equation for determining the constants $H(0,0)$ and $H(0,1)$. The second equation is obtained by putting $w=z$ in (2.5), dividing both sides by $z-1$ and subsequently substituting $z=1$. This yields

$$
\rho-1=H_{1}(1)\left(\frac{\mu_{\mathrm{fs}}}{\mu_{\mathrm{P}}}-1\right)-H(0,0)-\frac{\gamma}{\mu_{\mathrm{P}}} H(0,1) .
$$


Putting $z=1$ in (2.6) leads to the - obvious - relation

$$
\mu_{\mathrm{fs}} H_{1}(1)=\gamma H(0,1) \text {. }
$$

Combination of (2.17) and (2.18) gives us a second relation between $H(0,0)$ and $H(0,1)$,

$$
H(0,1)=\frac{\mu_{\mathrm{fs}}}{\gamma}(1-\rho-H(0,0))
$$

Introduce

$$
Q(z):=\sum_{i=0}^{\infty}\left\{C\left(\delta^{(i)}(z)\right) / \prod_{j=1}^{\infty} D\left(\delta^{(j)}(z)\right)\right\}, \quad|z| \leqslant 1
$$

combination of $(2.15),(2.16)$ and (2.19) then leads to our main result:

Theorem 1.

$$
\begin{aligned}
& H(0, z)=\sum_{m=0}^{\infty} \operatorname{Pr}\{\boldsymbol{x}=0, \boldsymbol{n}=m\} z^{m}=(1-\rho) \prod_{j=0}^{\infty} D\left(\delta^{(j)}(z)\right) \frac{Q(z)-Q\left(z_{1}\right)}{1\left(\gamma / \mu_{\mathrm{fs}}\right) Q\left(z_{1}\right)}, \quad|z| \leqslant 1 \\
& H(0,0)=\operatorname{Pr}\{\boldsymbol{x}=0, \boldsymbol{n}=0\}=\frac{1-\rho}{1-\left(\gamma / \mu_{\mathrm{fs}}\right) Q\left(z_{1}\right)}
\end{aligned}
$$

Finally, the joint generating function $\mathrm{H}(w, z)$ of $\boldsymbol{x}$ and $\boldsymbol{n}$ follows from (2.7) and Theorem 1.

Remark 3. In the special 'product-form case' $\mu_{\mathrm{P}}=\mu_{\mathrm{fs}}$, cf. Section 1 , it can easily be checked that substitution of

$$
H(0, z)=H(0,0) \frac{1}{1-\rho z}
$$

in (2.9) leads to an identity.

Remark 4. It follows from (2.16) and (2.20) that

$$
-Q\left(z_{1}\right)=\frac{H(0,0)}{H(0,1)}=\operatorname{Pr}\{\boldsymbol{n}=0 \mid \boldsymbol{x}=0\} \text {. }
$$

This interpretation implies in particular, cf. (2.22), that $H(0,0) \leqslant 1-\rho$ (cf. also (2.19)).

Remark 2 implies that, in order to numerically evaluate the infinite sum and product in, e.g., $Q\left(z_{1}\right)$, it is sufficient to take only a few terms of this sum and product - unless $\rho$ is close to one. Extensive numerical experiments confirm this. We have calculated $H(0,0)$ and $H(0,1)$ using.(2.22) and (2.19), replacing $Q\left(z_{1}\right)$ in (2.22) by

$$
Q^{(N)}\left(z_{1}\right):=\sum_{i=0}^{N}\left\{C\left(\delta^{(i)}\left(z_{1}\right)\right) / \prod_{j=1}^{N} D\left(\delta^{(j)}\left(z_{1}\right)\right)\right\} .
$$

The numerical calculations suggest the following. For $\rho \leqslant 0.6, N=5$ is already sufficient to calculate $H(0,0)$ and $H(0,1)$ with an error of at most $1 \%$; and for $N=10$, the error is already negligible. For $\rho \approx 0.75, N=10$ leads to an error of approximately $1 \%$; for $\rho \approx 0.9, N=20$ may still yield an error of $4 \%$. The numerical calculations are extremely simple, hardly occupying any computer time.

It is not difficult to evaluate various performance measures, starting from Theorem 1. Following Kaufman's [2] terminology, define $L_{\mathrm{fs}}$ and $L_{\mathrm{p}}$ to be the mean number of fs and Poisson customers, respectively, in $Q$ at a random epoch, and let $L=L_{\mathrm{fs}}+L_{\mathrm{p}}$. (2.19) and (2.22) enable us to evaluate 


$$
L_{\mathrm{fs}}=1-H(0,1)=\operatorname{Pr}\{\text { fs customer is in } Q\} .
$$

One can obtain the mean total queue length $L$ in $Q$ by performing some straightforward calculations, starting from the identity $L=\left.(\mathrm{d} / \mathrm{d} z) H(z, z)\right|_{z=1}$, or one can, as in [2], use basic relations such as Little's law to obtain

$$
L=L_{\mathrm{P}}+L_{\mathrm{fs}}=\left(\frac{\mu_{\mathrm{P}}}{\mu_{\mathrm{fs}}} L_{\mathrm{fs}}+1\right) \frac{\rho}{1-\rho}+L_{\mathrm{fs}} .
$$

Note that $L_{\mathrm{P}}=\rho /(1-\rho)$ in the case without finite source.

Introduce, for the fs customer, $\lambda_{\mathrm{fs}}, W_{\mathrm{fs}}, S_{\mathrm{fs}}, n_{\mathrm{fs}}^{(a)}$ and $n_{\mathrm{fs}}^{(d)}$, denoting respectively his arrival rate at $Q$, his mean waiting time and mean sojourn time at $Q$, the mean number of customers found by him in $Q$ upon arrival and left behind by him in $Q$ upon departure. All these quantities can be expressed in $L_{\mathrm{fs}}=1-H(0,1)$,

$$
\begin{aligned}
& \lambda_{\mathrm{fs}}=\gamma H(0,1)=\gamma\left(1-L_{\mathrm{fs}}\right), \\
& S_{\mathrm{fs}}=W_{\mathrm{fs}}+1 / \mu_{\mathrm{fs}}=L_{\mathrm{fs}} / \lambda_{\mathrm{fs}}=\frac{L_{\mathrm{fs}}}{\gamma\left(1-L_{\mathrm{fs}}\right)}, \\
& n_{\mathrm{fs}}^{(a)}=\mu_{\mathrm{p}} W_{\mathrm{fs}}=-\frac{\mu_{\mathrm{p}}}{\mu_{\mathrm{fs}}}+\mu_{\mathrm{p}} \frac{L_{\mathrm{fs}}}{\gamma\left(1-L_{\mathrm{fs}}\right)}, \\
& n_{\mathrm{fs}}^{(d)}=\lambda S_{\mathrm{fs}}=\lambda \frac{L_{\mathrm{fs}}}{\gamma\left(1-L_{\mathrm{fs}}\right)} .
\end{aligned}
$$

It is now easy to evaluate these performance measures numerically.

Remark 5. In all our numerical experiments $n_{\mathrm{fs}}^{(a)} \geqslant(\leqslant) n_{\mathrm{fs}}^{(d)}$ iff $\mu_{\mathrm{fs}} \geqslant(\leqslant) \mu_{\mathrm{p}}$, with equality iff $\mu_{\mathrm{fs}}=\mu_{\mathrm{p}}$. Kaufman's approximation [2] also predicts this result, which is intuitively obvious. Indeed, during an fs waiting time in $Q, Q$ behaves like an ordinary $M / M / 1$ queue (for which the queue length distributions at arrival and departure epochs are the same); but when $\mu_{\mathrm{fs}}>(<) \mu_{\mathrm{P}}$, the queue length in $Q$ during an fs service on the average builds up less (more) than it does during a service in an ordinary $M / M / 1$ queue.

The difference between $n_{\mathrm{fs}}^{(a)}$ and $n_{\mathrm{fs}}^{(d)}$ becomes negligible for relatively large values of $\gamma$ - which is obvious as in this case each fs departure from $Q$ is almost immediately followed by an fs arrival at $Q$.

Tables 1 through 6 display numerical results for $n_{\mathrm{fs}}^{(a)}, n_{\mathrm{fs}}^{(d)}, L_{\mathrm{p}}, L, \rho /(1-\rho)$ (the mean queue length in the model without finite source) and Kaufman's approximation $n_{\mathrm{fs}, \mathrm{K}}^{(a)}$ for $n_{\mathrm{fs}}^{(a)}$. In all six cases $\mu_{\mathrm{P}}=1$. The

\begin{tabular}{|c|c|c|c|c|c|c|}
\hline$\rho$ & $n_{f, \mathrm{~K}}^{(a)}$ & $n_{\mathrm{fs}}^{(a)}$ & $n_{f_{\mathrm{s}}}^{(d)}$ & $L$ & $L_{\mathrm{P}}$ & $\frac{\rho}{1-\rho}$ \\
\hline 0.15 & 0.54 & 0.37 & 0.81 & 1.15 & 0.63 & 0.18 \\
\hline 0.30 & 1.37 & 1.06 & 1.82 & 2.15 & 1.60 & 0.43 \\
\hline 0.45 & 2.76 & 2.31 & 3.29 & 3.84 & 3.25 & 0.82 \\
\hline 0.60 & 5.48 & 4.83 & 5.90 & 7.13 & 6.47 & 1.50 \\
\hline 0.75 & 12.17 & 11.22 & 12.16 & 15.23 & 14.47 & 3.00 \\
\hline 0.90 & 41.41 & 40.18 & 40.66 & 50.42 & 49.52 & 9.00 \\
\hline
\end{tabular}
six cases consider various combinations of relatively small and relatively large values of $\gamma$ and $\mu_{\mathrm{fs}}$.

Kaufman [2] approximates $L_{\mathrm{fs}}$ by introducing one basic approximation assumption, viz.,

$$
n_{\mathrm{fs}}^{(a)} \approx n_{\mathrm{fs}, \mathrm{K}}^{(a)}=\left(\left(\frac{\mu_{\mathrm{P}}}{\mu_{\mathrm{fs}}}-1\right) L_{\mathrm{fs}}+1\right) \frac{\rho}{1-\rho}
$$

Table 1

Mean queue lengths; $\gamma=0.2, \mu_{\mathrm{rs}}=0.2, \mu_{\mathrm{P}}=1$ 
Table 2

Mean queue lengths; $\gamma=0.2, \mu_{\mathrm{fs}}=1, \mu_{\mathrm{P}}=1$

\begin{tabular}{|c|c|c|c|c|c|c|}
\hline$\rho$ & $n_{\mathrm{fs}, \mathrm{K}}^{(a)}$ & $n_{\mathrm{fs}}^{(a)}$ & $n_{\mathrm{fs}}^{(d)}$ & $L$ & $L_{\mathrm{P}}$ & $\frac{\rho}{1-\rho}$ \\
\hline 0.15 & 0.18 & 0.18 & 0.18 & 0.40 & 0.21 & 0.18 \\
\hline 0.30 & 0.43 & 0.43 & 0.43 & 0.75 & 0.52 & 0.43 \\
\hline 0.45 & 0.82 & 0.82 & 0.82 & 1.30 & 1.04 & 0.82 \\
\hline 0.60 & 1.50 & 1.50 & 1.50 & 2.33 & 2.00 & 1.50 \\
\hline 0.75 & 3.00 & 3.00 & 3.00 & 4.78 & 4.33 & 3.00 \\
\hline 0.90 & 9.00 & 9.00 & 9.00 & 15.67 & 15.00 & 9.00 \\
\hline
\end{tabular}

Table 3

Mean queue lengths; $\gamma=0.2, \mu_{\mathrm{fs}}=9, \mu_{\mathrm{P}}=1$

\begin{tabular}{|c|c|c|c|c|c|c|}
\hline$\rho$ & $n_{\mathrm{fs}, \mathrm{K}}^{(a)}$ & $n_{\mathrm{fs}}^{(a)}$ & $n_{\mathrm{fs}}^{(d)}$ & $L$ & $L_{\mathrm{p}}$ & $\frac{\rho}{1-\rho}$ \\
\hline 0.15 & 0.17 & 0.15 & 0.04 & 0.23 & 0.18 & 0.18 \\
\hline 0.30 & 0.40 & 0.35 & 0.14 & 0.52 & 0.43 & 0.43 \\
\hline 0.45 & 0.72 & 0.64 & 0.34 & 0.96 & 0.83 & 0.82 \\
\hline 0.60 & 1.24 & 1.12 & 0.74 & 1.73 & 1.53 & 1.50 \\
\hline 0.75 & 2.19 & 2.07 & 1.64 & 3.41 & 3.10 & 3.00 \\
\hline 0.90 & 4.85 & 5.28 & 4.85 & 10.04 & 9.52 & 9.00 \\
\hline
\end{tabular}

Table 4

Mean queue lengths; $\gamma=9, \mu_{\mathrm{fs}}=0.2, \mu_{\mathrm{P}}=1$

\begin{tabular}{|c|c|c|c|c|c|c|}
\hline$\rho$ & $n_{\mathrm{fs}, \mathrm{K}}^{(a)}$ & $n_{\mathrm{fs}}^{(a)}$ & $n_{\mathrm{fs}}^{(d)}$ & $L$ & $L_{\mathrm{P}}$ & $\frac{\rho}{1-\rho}$ \\
\hline 0.15 & 0.87 & 0.84 & 0.88 & 2.02 & 1.04 & 0.18 \\
\hline 0.30 & 2.12 & 2.08 & 2.12 & 3.52 & 2.54 & 0.43 \\
\hline 0.45 & 4.05 & 4.01 & 4.05 & 5.85 & 4.86 & 0.82 \\
\hline 0.60 & 7.45 & 7.40 & 7.44 & 9.92 & 8.93 & 1.50 \\
\hline 0.75 & 14.93 & 14.89 & 14.92 & 18.91 & 17.92 & 3.00 \\
\hline 0.90 & 44.92 & 44.89 & 44.90 & 54.90 & 53.90 & 9.00 \\
\hline
\end{tabular}

Table 5

Mean queue lengths; $\gamma=9, \mu_{\mathrm{fs}}=1, \mu_{\mathrm{P}}=1$

\begin{tabular}{|c|c|c|c|c|c|c|}
\hline$\rho$ & $n_{\mathrm{fs}, \mathrm{K}}^{(a)}$ & $n_{\mathrm{fs}}^{(a)}$ & $n_{\mathrm{fs}}^{(d)}$ & $L$ & $L_{\mathrm{P}}$ & $\frac{\rho}{1-\rho}$ \\
\hline 0.15 & 0.18 & 0.18 & 0.18 & 1.25 & 0.34 & 0.18 \\
\hline 0.30 & 0.43 & 0.43 & 0.43 & 1.75 & 0.83 & 0.43 \\
\hline 0.45 & 0.82 & 0.82 & 0.82 & 2.53 & 1.59 & 0.82 \\
\hline 0.60 & 1.50 & 1.50 & 1.50 & 3.89 & 2.94 & 1.50 \\
\hline 0.75 & 3.00 & 3.00 & 3.00 & 6.89 & 5.92 & 3.00 \\
\hline 0.90 & 9.00 & 9.00 & 9.00 & 18.89 & 17.90 & 9.00 \\
\hline
\end{tabular}

(with $L_{\mathrm{fs}}$ yet to be determined). Comparison of $n_{\mathrm{fs}}^{(a)}$ and $n_{\mathrm{fs}, \mathrm{K}}^{(a)}$ shows the accuracy of Kaufman's approximation assumption. The approximation is exact when $\mu_{\mathrm{fs}}=\mu_{\mathrm{p}}$ (cf. Tables 2 and 5), and it is very accurate when $\gamma$ is relatively large (Table 3). Comparison of $n_{\mathrm{fs}}^{(a)}$ and $n_{\mathrm{fs}}^{(d)}$ confirms Remark 5 above concerning their relative order.

In Tables 2 and 5 equality of $\mu_{\mathrm{fs}}$ and $\mu_{\mathrm{P}}$ leads to $n_{\mathrm{fs}}^{(a)}=n_{\mathrm{fs}}^{(d)}=\rho /(1-\rho)$. In this case we have, cf. 
Table 6

Mean queue lengths; $\gamma=9, \mu_{\mathrm{fs}}=9, \mu_{\mathrm{p}}=1$

\begin{tabular}{|c|c|c|c|c|c|c|}
\hline$\rho$ & $n_{\mathrm{fs}, \mathrm{K}}^{(a)}$ & $\overline{n_{\mathrm{fs}}^{(a)}}$ & $n_{\mathrm{fs}}^{(d)}$ & $L$ & $L_{\mathrm{P}}$ & $\frac{\rho}{1-\rho}$ \\
\hline 0.15 & 0.09 & 0.04 & 0.02 & 0.76 & 0.19 & 0.18 \\
\hline 0.30 & 0.19 & 0.08 & 0.06 & 1.10 & 0.46 & 0.43 \\
\hline 0.45 & 0.30 & 0.16 & 0.12 & 1.59 & 0.88 & 0.82 \\
\hline 0.60 & 0.46 & 0.28 & 0.24 & 2.41 & 1.63 & 1.50 \\
\hline 0.75 & 0.72 & 0.55 & 0.50 & 4.14 & 3.29 & 3.00 \\
\hline 0.90 & 1.50 & 1.57 & 1.51 & 10.88 & 9.94 & 9.00 \\
\hline
\end{tabular}

Kaufman [2],

$$
L=2 \frac{\rho}{1-\rho}+\frac{\hat{\rho}-\rho}{1+\hat{\rho}-\rho} ;
$$

here $\hat{\rho}=\gamma / \mu_{\mathrm{P}}$. Hence the mean queue length at an arbitrary epoch can be much higher than the mean queue length at an arrival or departure epoch from the fs customer - as is confirmed by the tables.

Finally, comparison of $L_{\mathrm{p}}$ and $\rho /(1-\rho)$ shows the influence of the one fs customer on the mean queue length of the Poisson customers. This influence is small for small values of $\gamma$ and large values of $\mu_{\mathrm{fs}}$ (cf. Table 3), but very pronounced for large values of $\gamma$ and small values of $\mu_{\mathrm{fs}}$ (cf. Table 4).

\section{Asymptotics}

In this section we study the asymptotic behavior of $h(0, m)$ for $m \rightarrow \infty$. In the special case $\gamma=0$ the fs customer does not influence the service of the Poisson stream of customers, and $M / M / 1$ theory yields

$$
H(0, z)=(1-\rho) /(1-\rho z)
$$

hence

$$
h(0, m)=(1-\rho) \rho^{m}, \quad m=0,1, \ldots,
$$

and trivially we see that asymptotically $h(0, m) \sim \rho^{m}$.

In the following let $\gamma>0$. We want to investigate the influence of the fs customer on the asymptotic behavior of $h(0, m)$, by exploiting our knowledge of $H(0, z)$ (cf. (2.21)).

Theorem 2. For $\gamma>0$ fixed,

$$
h(0, m) \sim\left\{\max \left[\rho, \lambda /\left(\lambda+\mu_{\mathrm{fs}}\right)\right]\right\}^{m}, \quad m \rightarrow \infty .
$$

Proof. The pole of $H(0, z)$ with the smallest absolute value will determine the asymptotic behavior of $h(0, m)$ for $m \rightarrow \infty$; if this pole is $x$, then $h(0, m) \sim(1 / x)^{m}, m \rightarrow \infty$. Starting-point is expression (2.21) for $H(0, z)$. First consider the poles of $C(z)$ and $D(z)$, rewriting (2.10) and (2.11),

$$
\begin{aligned}
& C(z)=\frac{(1 / \rho)(1-z)}{\left(z-z_{1}\right)\left(z-z_{2}\right)}, \\
& D(z)=\frac{\gamma \mu_{\mathrm{fs}} z / \lambda^{2}}{\left(z-z_{1}\right)\left(z-z_{2}\right)\left(z-z_{3}\right)} .
\end{aligned}
$$

Here $z_{1}$ and $z_{2}$ are the two zeros of $z\left[\lambda(1-z)+\mu_{\mathrm{P}}(1-1 / z)+\gamma\right] ; z_{1}$ has been defined in (2.12), where it was observed that $0<z_{1}<1$, and

$$
\begin{aligned}
& z_{2}=\left[\lambda+\mu_{\mathrm{P}}+\gamma+\sqrt{\left(\lambda+\mu_{\mathrm{P}}+\gamma\right)^{2}-4 \lambda \mu_{\mathrm{P}}}\right] / 2 \lambda=1 / z_{1 \rho}>1 / \rho>1, \\
& z_{3}=\left(\lambda+\mu_{\mathrm{fs}}\right) / \lambda>1 .
\end{aligned}
$$


We not only have to consider these poles $z_{1}, z_{2}, z_{3}$; we also have to consider the points $z$ for which an $n \in\{1,2, \ldots\}$ exists, such that $\delta^{(n)}(z)$ equals $z_{1}$ or $z_{2}$ or $z_{3}$. Introduce the inverse $e(\cdot)$ of $\delta(\cdot)$ : for $|z| \leqslant 1$,

$$
\begin{aligned}
& e(z):=e^{(1)}(z):=1+1 / \rho-1 / \rho z, \\
& e^{(n)}(z):=e\left(e^{(n-1)}(z)\right), \quad n=2,3, \ldots .
\end{aligned}
$$

Now we consider in turn the singularities $e^{(n)}\left(z_{1}\right), e^{(n)}\left(z_{2}\right), e^{(n)}\left(z_{3}\right), n=0,1, \ldots$ (with $e^{(0)}(z) \equiv z$ ) of the expression for $H(0, z)$ in (2.21). It is clear that $e^{(n)}\left(z_{1}\right), n=0,1, \ldots$, is a removable singularity of $H(0, z)$.

Next consider $e^{(n)}\left(z_{2}\right), n=0,1, \ldots$. The fact that $z_{2}>1 / \rho$ implies that

$$
1 / \rho<e^{(n)}\left(z_{2}\right)<1+1 / \rho, \quad n=1,2, \ldots,
$$

and (cf. Lemma 1),

$$
\frac{1-e^{(n-1)}\left(z_{2}\right)}{1-e^{(n)}\left(z_{2}\right)}=\rho e^{(n-1)}\left(z_{2}\right)>1,
$$

so

$$
e^{(n)}\left(z_{2}\right)<e^{(n-1)}\left(z_{2}\right) ;
$$

for $n \rightarrow \infty$, it follows that

$$
e^{(n)}\left(z_{2}\right) \downarrow 1 / \rho .
$$

Finally consider $e^{(n)}\left(z_{3}\right), n=0,1, \ldots$ If $\lambda+\mu_{\mathrm{fs}}>\mu_{\mathrm{p}}$, then $z_{3}>1 / \rho$, and $e^{(n)}\left(z_{3}\right) \downarrow 1 / \rho$ for $n \rightarrow \infty$. If $\lambda+\mu_{\mathrm{fs}}=\mu_{\mathrm{P}}$, then $e^{(n)}\left(z_{3}\right)=1 / \rho$ for $n=0,1, \ldots$ If $\lambda+\mu_{\mathrm{fs}}<\mu_{\mathrm{p}}$, then $e^{(n)}\left(z_{3}\right)>e^{(n-1)}\left(z_{3}\right)>\ldots>z_{3}=$ $\left(\lambda+\mu_{\mathrm{fs}}\right) / \lambda$; only in this last case $z_{3}$ is the pole of $H(0, z)$ with smallest absolute value. This concludes the proof.

Remark 6. It can easily be derived from Theorem 2 and formula (2.7) with $w=z$, that, globally speaking, $\operatorname{Pr}\{\boldsymbol{n}=m\}$ has a similar asymptotic behavior as $\operatorname{Pr}\{\boldsymbol{x}=0, \boldsymbol{n}=m\}$. The interpretation of Theorem 2 is the following. For $\lambda<\mu_{\mathrm{P}}<\lambda+\mu_{\mathrm{fs}}$, the Poisson customers dominate the queue length behavior at $Q$. For larger values of $\mu_{\mathrm{P}}$ this domination disappears. Indeed, in the limiting case $\mu_{\mathrm{P}} \rightarrow \infty$ the queue at $Q$ can only build up during service times of the fs customer at $Q$. In this respect the term $\left\{\lambda /\left(\lambda+\mu_{\mathrm{fs}}\right)\right\}^{m}$ is significant, as it denotes the probability of $m$ Poisson arrivals during one fs service in $Q$.

Note

During the preparation of this manuscript it came to the author's attention that Dr. B.T. Doshi and Dr. W.S. Wong of AT\&T Bell Laboratories recently have also analysed the model under consideration [1]. Their approach is different, and they obtain the generating function of the queue length distribution at $Q$ seen by the arriving $f_{s}$ customer (which is different from $H(0, z)$, but which also yields $L_{\mathrm{fs}}$ and $S_{\mathrm{fs}}$ ). They also consider the case of LIFO service in $Q$.

In a future study we shall determine the joint distribution of queue length at $Q$ and position of the fs customer at departure epochs from $Q$, for completely general service time distributions in $Q$; this will enable us to obtain the waiting time and sojourn time distributions of the fs customer in $Q$.

\section{Acknowledgment}

The author is indebted to Professor J.W. Cohen for stimulating discussions. 


\section{References}

[1] B.T. Doshi and W.S. Wong, "Exact solution to a simple finite infinite source interaction model", Report, AT\&T Bell Laboratories, Holmdel, NJ, 1985, forthcoming in: Queueing Systems: Theory and Applications.

[2] J.S. Kaufman, "Finite and infinite source interactions", in: E. Gelenbe, ed., Performance '84, North-Holland, Amsterdam, 1984, 345-359.

[3] L. Takacs, "Two queues attended by a single server", Op. Research 16, 639-650 (1968).

[4] B. Vinod and J.J. Solberg, "Performance models for unreliable flexible manufacturing systems", OMEGA Int. J. of Mgmt. Sci. 12, 299-308 (1984). 